\title{
Photografted methacrylate-based monolithic columns coated with cellulose tris(3,5- dimethylphenylcarbamate) for chiral separation in CEC
}

\author{
Romina Noel Echevarría ${ }^{1}$, Enrique Javier Carrasco-Correa ${ }^{2}$, Sonia Keunchkarian ${ }^{1}$, \\ Mario Reta ${ }^{1}$, José Manuel Herrero-Martinez ${ }^{2 *}$ \\ ${ }^{1}$ Laboratorio de Investigación y Desarrollo de Métodos Analíticos (LIDMA), Facultad de \\ Ciencias Exactas, 49 y 115 (1900). Universidad Nacional de La Plata, CIC-PBA, Argentina \\ ${ }^{2}$ Department of Analytical Chemistry, University of Valencia, Dr. Moliner 50, \\ 46100 Burjassot, Valencia, Spain
}

Running title: Photografted polymeric monoliths with CDMPC coating for chiral separations in $\mathrm{CEC}$

*Corresponding author: Dr. José Manuel Herrero Martínez

$$
\begin{aligned}
& \text { Tel: +34963544062 } \\
& \text { Fax: +34963544436 } \\
& \text { e-mail: jmherrer@uv.es }
\end{aligned}
$$

Abbreviations used in the text: CDs - cyclodextrins; CDMPC - cellulose tris(3,5dimethylphenylcarbamate); CSP - chiral stationary phase; META- [2(methacryloyloxy)ethyl] trimethylammonium chloride

Keywords: Capillary electrochromatography; cellulose tris(3,5-dimethylphenylcarbamate); chiral monolithic organic column; enantioseparation; photografting

Received: 10 23, 2017; Revised: 01 20, 2018; Accepted:01 20, 2018

This article has been accepted for publication and undergone full peer review but has not been through the copyediting, typesetting, pagination and proofreading process, which may lead to differences between this version and the Version of Record. Please cite this article as doi: 10.1002/jssc. 201701234 . 


\section{Abstract}

A chiral capillary monolithic column for enantiomer separation in capillary electrochromatography was prepared by coating cellulose tris(3,5-dimethylphenylcarbamate) on porous glycidyl methacrylate-co-ethylene dimethacrylate monolith in capillary format grafted with chains of [2(methacryloyloxy)ethyl] trimethylammonium chloride. The surface modification of the monolith by the photografting of [2(methacryloyloxy)ethyl] trimethylammonium chloride monomer as well as the coating conditions of cellulose tris(3,5dimethylphenylcarbamate) onto the grafted monolithic scaffold were optimized to obtain a stable and reproducible chiral stationary phase for capillary electrochromatography. The effect of organic modifier (acetonitrile) in aqueous mobile phase for the enantiomer separation by capillary electrochromatography was also investigated. Several pairs of enantiomers including acidic, neutral, and basic analytes were tested and most of them were partially or completely resolved under aqueous mobile phases. The prepared monolithic chiral stationary phases exhibited a good stability, repeatability, and column-to-column reproducibility, with relative standard deviations below $11 \%$ in the studied electrochromatographic parameters.

\section{Introduction}

The development of enantioseparation methodologies has received great attention during the past several decades, particularly in the pharmaceutical and environmental fields $[1,2]$. Within the enantioseparation techniques, HPLC using chiral stationary phases (CSPs) has been the most popular one for analyzing chiral compounds. In the last decade, the miniaturization of separations has attracted an increasing interest due to its advantages such as low consumption of sample, solvents and stationary phases. Thus, most of the chiral selectors successfully used in conventional HPLC have been transferred to small-scale 
separation techniques such as capillary/nano LC, CE and CEC [1-7]. In particular, electrodriven techniques have demonstrated higher efficiency over pressure-driven systems due to the flat flow profile of EOF, which significantly reduces the band-broadening by eddy diffusion. Furthermore, the use of bonded CSPs in CEC columns has showed an excellent enantiomeric selectivity and sample loading capacity compared to conventional open-tubular CE capillaries $[2,8,9]$.

At present, several types of chiral CEC columns have been described, including opentubular, particle-packed and monolithic capillary columns. Monolithic stationary phases present some benefits over the open-tubular and packed columns. Thus, the monolithic supports possess higher loading capacities than open-tubular capillaries due to its larger phase ratio. Besides, monolithic capillary columns have a singular porous structure, easy to prepare, and do not require retaining frits, thus avoiding plugging or formation of bubbles during CEC operation.

Monolithic stationary phases can be classified into two general categories, silica- and organic polymer-based monoliths. Several chiral selectors (e.g. cyclodextrins (CDs), chiral ion-exchangers, proteins, cellulose derivatives, etc) have been incorporated into silica-based monoliths for enantioseparation purposes [10-12]. However, these monolithic columns showed a limited chemical stability over the $\mathrm{pH}$ range compared to the organic polymer counterparts. Other important feature of these polymeric organic monoliths is its readily modifiable surface chemistry, which make these materials promising supports to prepare CSPs with different functional groups.

In spite of these favorable attributes, few studies have focused on the preparation of CSPs based on organic polymer monoliths for CEC. Thus, the immobilization of diverse chiral selectors such as CDs [13], proteins [3], macrocyclic antibiotics [14], crown ethers 
$[15,16]$ and ligand-exchange reagents $[17,18]$ onto the polymethacrylates and polyacrylamide rods have been reported [7].

Polysaccharide derivatives (e.g. cellulose tris(3,5-dimethylphenylcarbamate) (CDMPC)) are often considered as one of the most successful chiral selectors due to their broad enantioselectivity, outstanding efficiency and stability [19]. In CEC, these CSPs have been prepared for enantioseparations by coating on or bonding to particulate packed columns [20-22] or silica-based monolithic beds $[1-3,11,12]$. In particular, the coating approach could be accomplished on the basis of multiple hydrogen-bonding sites present in these supports, which allow to tightly retain to the polysaccharide derivatives. However, few studies [9] have been reported on the use of cellulose derivatives, such as CDMPC, for the preparation of polymer monoliths in CEC.

As we mentioned above, different stationary phase chemistries can be easily obtained by post-polymerization reactions on the surface of the polymeric-based monoliths. Within these strategies, surface grafting constitutes a very versatile tool to prepare a wide variety of surface chemistries from a single "parent" monolith [23-25]. Thus, Eeltink et al. co-grafted [(2-methacryloyloxy)ethyl] trimethylammonium chloride (META) and butyl acrylate to control both EOF and hydrophobicity in CEC columns [26]. Hilder et al. [27] prepared stationary phases by photografting of two layers of polymer chains (first layer consisted of 2acryloamido-2-methyl-1-propanesulfonic acid, followed by a "covering" layer of hydrophobic butyl acrylate) for the rapid and efficient CEC separation of peptides. Also, grafted monolithic columns have been developed for biomolecule immobilization [28]. However, to the best of our knowledge, the use of grafted platforms with coated polysaccharide derivatives as chiral sorbents has not yet been explored. 
In this work, glycidyl methacrylate-based monoliths (synthesized by UV irradiation) were photografted with a positively charged monomer (META) to incorporate quaternary ammonium groups into a monolithic matrix thus providing a high number of hydrogen bond sites. The grafting (META content in the graft solution) as well as the coating conditions (concentration of CDMPC solution) were investigated to achieve CSPs with enhanced enantioseparation performance. The effect of the mobile phase composition on enantiomeric separation (retention and resolution) was also investigated. To evaluate the chiral capacity of the prepared monolithic CSPs, several acidic, basic and neutral racemates were tested. Additionally, the reproducibility and stability of these CSPs were evaluated.

\section{Materials and methods}

\subsection{Reagents and materials}

Microcrystalline cellulose (Avicel ${ }^{\circledR} \mathrm{PH}$ 101) was purchased from Merck (Darmstadt, Germany). Anhydrous pyridine, 3,5-dimethylphenylisocyanate, glycidyl methacrylate (97\%, GMA), ethylene glycol dimethacrylate $\quad(98 \%, \quad$ EDMA), [2(methacryloyloxy)ethyl]trimethylammonium chloride $(75 \%$ in water, META), benzophenone, cyclohexanol (99\%), 1-dodecanol (99\%), 3-(trimethoxysilyl) propyl methacrylate (98\%) and Lugol's (iodine/potassium iodide) solution from Sigma-Aldrich (St. Louis, MO, USA). $\alpha, \alpha^{\prime}$-Azobisisobutyronitrile (AIBN) from Fluka (Buchs, Switzerland) was used as the radical initiator. HPLC-grade acetonitrile (ACN), methanol (MeOH), acetone, acetic acid (HAcO) and phosphate acid from VWR (Radnor, Boston, MA, USA), were also employed. Deionized water was obtained with a Barnstead deionizer (Sybron, Boston, MA, USA).

Uracil and racemic compounds of trans-stilbene oxide, benzoin, 2-phenyl-1-propanol, Tröger's base, mefloquin, propranolol, naproxen, ibuprofen and phenylalanine were 
purchased from Sigma-Aldrich. The chemical structures of the tested analytes are presented in Fig. S1.

Uncoated fused-silica capillaries of $33.5 \mathrm{~cm}$ total length and $365 \mu \mathrm{m}$ O.D. $\times 100 \mu \mathrm{m}$ I.D. with a UV-transparent external coating (Polymicro Technologies, Phoenix, AZ, USA) were used. The effective monolithic bed length was $25 \mathrm{~cm}$.

\subsection{Instrumentation}

An EXETER CE 440 CHN elemental analyzer (CE Instruments, Milan, Italy) was used for elemental analysis of synthesized CDMPC. FTIR spectra of this chiral selector and its pristine material were obtained with a Nicolet 380 spectrophotometer fitted with a single reflection attenuated total reflectance (ATR) accessory. Spectra were accomplished from 4000 to $500 \mathrm{~cm}^{-1}$ at a $2 \mathrm{~cm}^{-1}$ resolution with 50 scans. An irradiation chamber (model CL1000) from UVP (Upland, CA, USA), equipped with UV lamps $(5 \times 8 \mathrm{~W}, 254 \mathrm{~nm})$, was used for the preparation and photografting of the monolithic columns. A syringe pump (Model 100, KD Scientific, New Hope, PA, USA) was employed to introduce the functionalizing reagents into the monolithic capillary columns. An HPLC pump (1100 series, Agilent Technologies, Waldbronn, Germany) was used for washing the prepared monolithic columns.

CEC experiments were performed on a $\mathrm{HP}^{3 \mathrm{D}} \mathrm{CE}$ instrument (Agilent) equipped with a diode-array UV detector and pressurized at both capillary ends with an external nitrogen supply. Data acquisition was performed with the ChemStation Software (Rev.A.10.01, Agilent). Before use, all mobile phases for CEC were degassed by ultrasonication. 
2.3. Synthesis and characterization of cellulose tris(3,5-dimethylphenylcarbamate)

The CDMPC was synthesized as described previously [29,30]. Briefly, dried microcrystalline cellulose $(1 \mathrm{~g})$ was refluxed in anhydrous pyridine $(30 \mathrm{~mL})$ for $8 \mathrm{~h}$. After cooling the mixture to room temperature, 3,5-dimethylphenyl isocyanate ( $3.3 \mathrm{~g})$ was added and allowed to react at $80^{\circ} \mathrm{C}$ for $24 \mathrm{~h}$ under stirring. Then, the reaction mixture, a dark amber viscous liquid, was cooled to room temperature. The solution was poured into $\mathrm{MeOH}$ (250 $\mathrm{mL}$ ) under vigorous stirring for $2 \mathrm{~h}$. The resulting white solid was collected by vacuum filtration and washed several times with $\mathrm{MeOH}$. The product was dried in air, and then under vacuum to constant weight.

The CDMPC was then characterized by elemental analysis to confirm the presence of the phenyl carbamate group. The results were as follows: $\mathrm{C}$ at $64.54 \%, \mathrm{H}$ at $6.08 \%$ and $6.77 \% \mathrm{~N}$ (calculated: $65.44 \% \mathrm{C}, 6.49 \% \mathrm{H}$, and $6.94 \% \mathrm{~N}$ ). The reaction yield calculated was 87\%. IR measurements of CDMPC were also accomplished, giving the following characteristic absorption bands: $v(\mathrm{C}=\mathrm{O}) 1750 \mathrm{~cm}^{-1}, v(\mathrm{~N}-\mathrm{H}) 3310 \mathrm{~cm}^{-1}, v$ (phenyl) $1602 \mathrm{~cm}^{-1}$. [2]. Also, absorption at $3500 \mathrm{~cm}^{-1}$ due to $-\mathrm{OH}$ groups corresponding to the native cellulose was slightly evidenced in the derivatized chiral selector (Fig. S2).

\subsection{Preparation of parent organic monoliths}

For the preparation of the polymeric monoliths in capillary format, the inner wall of the fused-silica capillaries was modified before polymerization, to provide covalent attachment of the monolith. For this purpose, wall modification with 3-(trimethoxysilyl) propyl methacrylate was performed as described previously $[31,32]$. The polymerization mixtures were prepared by weighing GMA (20 wt\%), EDMA (5 wt \%), and a binary poreforming solvent constituted by cyclohexanol $(70 \mathrm{wt} \%)$ and 1-dodecanol (5 wt $\%)$. AIBN (1 
wt $\%$ with respect to the monomers) was added as initiator [33]. After mixing, the polymerization mixture was sonicated for 10 min to obtain a clear solution and then purged with $\mathrm{N}_{2}$ for $10 \mathrm{~min}$. The preconditioned (silanized) capillary was filled with the polymerization mixture up to a length of $25 \mathrm{~cm}$. Photopolymerization was accomplished by irradiation of the capillaries within the UV chamber at $0.9 \mathrm{~J} \mathrm{~cm}^{-2}$ for $15 \mathrm{~min}$. Then, an HPLC pump was used to flush the columns for $60 \mathrm{~min}$ with $\mathrm{MeOH}$ to remove the pore-forming solvents and any possible unreacted monomers.

\subsection{Functionalization of polymeric monoliths by photografting}

The post-polymerization functionalization of the parent monoliths (GMA-based monoliths) was performed using a two-step approach as described previously in the literature $[34,35]$. Thus, the columns were flushed with a solution of benzophenone as a photoinitiator ( $5 \mathrm{wt} \%$ in $\mathrm{MeOH}$ ) at $0.06 \mathrm{~mL} \mathrm{~h}^{-1}$ for $1 \mathrm{~h}$ followed by $\mathrm{UV}$ irradiation at $1 \mathrm{~J} \mathrm{~cm}^{-2}$ for $10 \mathrm{~min}$. After the initiator was attached to the surface, the monolithic columns were flushed with $\mathrm{MeOH}$ to remove any unreacted benzophenone. Then, a solution of META (prepared at different percentages in $\mathrm{MeOH}$ ) was flushed at $0.06 \mathrm{~mL} \mathrm{~h}^{-1}$ for $1 \mathrm{~h}$. Next, the column ends were sealed, and the capillary was irradiated under the same conditions as above and the monoliths were finally washed with $\mathrm{MeOH}$.

\subsection{In situ coating of methacrylate monolith with cellulose tris(3,5-} dimethylphenylcarbamate)

The photografted monoliths were first flushed with acetone. Afterwards, the syringe pump was used to pump dispersions containing several amounts $\left(6-60 \mathrm{mg} \mathrm{mL}^{-1}\right)$ of CDMPC 
in acetone through the monoliths at a flow rate of $0.06 \mathrm{~mL} \mathrm{~h}^{-1}$ for $2 \mathrm{~h}$. Then, an HPLC pump was used to flush the columns with mobile phase for $60 \mathrm{~min}$.

\subsection{CEC conditions}

Before use, all mobile phases were degassed by ultrasonication for all CEC experiments. The monolithic column, placed in the CEC instrument was equilibrated with the mobile phase by progressively increasing the applied voltage from 5 to $10 \mathrm{kV}$ until a constant current and a stable baseline were achieved. Separations were performed at $25^{\circ} \mathrm{C}$. In all cases, the inlet and outlet vials were pressurized at $1 \mathrm{MPa}$ with nitrogen. All analytes were dissolved in the running mobile phase to give final concentrations ranged from 0.5 to $1.0 \mathrm{mg} \mathrm{mL}^{-1}$. The test mixtures were injected electrokinetically under $5 \mathrm{kV}$ for $3 \mathrm{~s}$. Detection was performed at 214 and $254 \mathrm{~nm}$. Specific separation conditions for different test analytes are indicated in the corresponding figure captions. The EOF rate was determined using uracil as the unretained marker.

\section{Results and discussion}

\subsection{Preparation of chiral monolithic columns. Influence of coating conditions of} cellulose tris(3,5-dimethylphenylcarbamate) on enantiomer separation

A careful control of the monolithic stationary phase morphology is an important issue to achieve a robust generic porous material that provides both enough surface area for the chiral selector coating and low flow resistance. For this purpose, photo-polymerized GMAbased monoliths were prepared as parent supports based on previous works [33-34]. The monoliths made under these conditions showed a high permeability due to the large flowthrough pores of around $1-3 \mu \mathrm{m}$ in size (Fig. 2A), and high permeability $\left(4.0 \cdot 10^{-14} \mathrm{~m}^{2}\right)$, which enormously facilitated the subsequent flushing steps used in the surface modification 
protocols.

In $\mathrm{CEC}$, the generation of the EOF is necessary to drive the mobile phase through the capillary column. To achieve a proper CEC separation of enantiomeric compounds, a strong anodic or cathodic EOF is required. Then, the monolith can be modified to incorporate ionizable groups. For this purpose, we used a two-stage photografting protocol, where the poly(GMA-co-EDMA) was modified with a positively charged monomer (META) to incorporate quaternary ammonium functionalities into a monolithic matrix. In this procedure, benzophenone is commonly used as the photoactive component for the initiation of photografting process of monolithic surfaces [37]. Hence, the conditions used for the first step (benzophenone) were taken from literature [37,38], whereas the initial conditions used for monomer grafting were as follows: $1.5 \mathrm{wt} . \%$ aqueous META solution at irradiation time of $10 \mathrm{~min}$.

The CDMPC loading is an important factor that influences on the column performance and its chiral selectivity. Under these experimental conditions, the coating of CDMPC onto the methacrylate monolith was conducted. Two variables, coating time and concentration of CDMPC solution, were investigated to obtain stable and reproducible CSPs. Solutions containing different amounts of CDMPC (up to $60 \mathrm{mg} \mathrm{mL}^{-1}$ ) dissolved in acetone were then prepared., In spite of the high viscosity of the polysaccharide solutions, the capillaries were properly rinsed due to the high permeability of the monolith. In any case, the coating time was long enough to achieve an appropriate surface coating with CDMPC. For this purpose, the starting coating time was considered when the drops (collected at the end of the column) containing the chiral selector gave a positive Lugol's assay. Our results suggest that a flushing time of $60 \mathrm{~min}$ was adequate to achieve a CDMPC coating through the monolithic matrix that allowed good column-to-column reproducibilities (see below). SEM 
image of the META-grafted monolith coated with CDMPC (Fig. 2B) was also taken, showing a slight increase in the globule size compared to the parent monolith (Fig. 2A). In addition, the permeability of the META-grafted monolith before and after coating with CDMPC was also measured, and no significant change (below $5 \%$ at $60 \mathrm{mg} \mathrm{mL}^{-1}$ ) was found. Next, several concentrations of CDMPC (from 6 to $60 \mathrm{mg} \mathrm{mL} L^{-1}$ ) in the coating solution were studied for CEC enantioseparation. The electrochromatographic performance of the columns prepared with different CDMPC contents was evaluated using Tröger's base as test analyte (Table 1). Regarding retention factor $(k)$, by increasing the amount of CDMPC coated onto surface monolith up to $15 \mathrm{mg} \mathrm{mL}^{-1}$, the $k$-values increased. This enhanced retention is suggested to be due to the increase of CDMPC content. However, a rise in the amount of CDMPC (from 15 to $60 \mathrm{mg} \mathrm{mL}^{-1}$ ) into the polymer system caused certain reduction in the $k$-values. These results may be due to different conformation or orientation of CDMPC on the META-grafted monolith surface induced by a change in the CDMC concentration used in the coating solution. Thus, at low CDMPC contents, each polymer chain may exist separately, whereas at high contents, an association and ordering of the CDMPC chains could result. It could provide an enhanced perfusion phenomena giving as a result changes in double-layer and a reduction in the retention times of Tröger's base. Moreover, it can be observed that an increase in the CDMPC solution concentration onto the monolithic support led to a progressive increase in enantioselectivity $(\alpha)$ and enantioresolution (Rs) values. However, column efficiency decreased as coating concentration of CDMPC was raised. Similar findings have been reported for silica-based monoliths coated with this chiral selector or other polysaccharide derivatives [39]. This behavior was attributed to a lower mass transfer between the mobile and the stationary phases $[1,40]$. In any case, the highest chiral resolution was achieved with a coating concentration of 
$60 \mathrm{mg} \mathrm{mL}^{-1}$ of CDMPC, and consequently, it was selected for further studies.

On the other hand, in all tested capillary columns, the immobilization of CDMPC onto the monolithic matrix was probably accomplished on the basis of strong hydrogen-bonding and electrostatic interactions between the polysaccharide derivative (resonance structures of carbamate moieties) and the META groups in the grafted surface of the polymeric network [9]. Other interaction contributions such as van der Waals interactions (due to phenyl moieties of CDMPC) might also contribute (although in a less extent) to this immobilization. To increase the interaction sites and consequently to enhance the amount of accessible CDMPC onto monolith surface, the META content present on the polymethacrylate-based monoliths was then investigated. For this purpose, the concentration of this monomer was varied, by keeping constant the irradiation time and performing the CDMPC coating step described above.

Previous to the CDMPC coating, the effect of concentration of META on the EOF was examined. As expected, a significant increase in EOF for monoliths grafted using solutions from 1.5 to 7.5 wt.\% was observed. However, high concentrations of META (15 $\mathrm{wt} \%$ ) led to a decrease in EOF content. It could be explained by a self-screening effect produced by the large concentration of META during photo-grafting, leaving a low available energy for the photo-initiator, which resulted in a lower concentration of available energetic free radicals and thus less grafted monomer onto the surface $[23,24]$. On the other hand, with increasing META content coated with CDMPC at the selected conditions, a reduction in EOF values was observed (up to $5.4 \cdot 10^{-9} \mathrm{~m}^{2} \mathrm{~V}^{-1} \mathrm{~s}^{-1}$ for monoliths grafted with $15 \mathrm{wt} \%$ of META solution). This decrease in the anionic EOF could be due to the excess of CDMPC, which reduced the available positively charged ammonium groups present on the monolith surface. In addition, the effect of the concentration of META on enantiomeric separation was also 
evaluated. As shown in Fig. 2 , an increase in the resolution and selectivity values were obtained from 1.5 to $7.5 \mathrm{wt} \%$ META content (see traces $\mathrm{A}$ and $\mathrm{B}$ ), whereas, at higher concentrations of grafted monomer $(15 \mathrm{wt} \%)$, a reduction in resolution was evidenced. The lower chromatographic efficiencies and resolution observed for the columns grafted with the largest META content could be the result of slow diffusion of the solutes within the chiral selector. At sight of these results, monoliths photografted with $7.5 \mathrm{wt} \%$ of META solution were selected for the following experiments.

\subsection{Evaluation of enantioseparation performance of chiral stationary phases}

The enantioseparation performance is influenced by the content of the organic modifier in the mobile phase. Thus, we studied the impact of ACN as organic modifier on the retention, resolution, and efficiency in the monolithic columns photografted with $7.5 \mathrm{wt} \%$ META and subsequently coated with $60 \mathrm{mg} \mathrm{mL}^{-1}$ CDMPC. Fig. 3 shows the effect of ACN variation on enantioseparation of Tröger's base. The results showed that retention factor and resolution decreased with increasing $\mathrm{ACN}$ content in mobile phase from 30 to $40 \%$. This trend suggested a hydrophobic interaction, typical in reversed-phase separations, between the CDMPC selector and chiral analytes $[9,41,42]$. As it can be seen, the best compromise between enantioseparation and analysis time was achieved with $35 \% \mathrm{ACN}$ in the mobile phase, and it was selected for further experiments.

To evaluate the separation ability of the prepared CDMPC coated polymethacrylate monolithic capillary columns, several acidic, basic, and neutral racemates were tested using aqueous mobile phases with different $\mathrm{pH}$ values. The obtained electrochromatographic data are given in Table 2, along with the separation conditions applied. Most of the racemates were successfully resolved, and the Rs values of enantiomers varied from $<0.5$ to 4.20. Electrochromatograms of some of the studied racemates are illustrated in Fig. 4. 
A comparison in terms of chiral recognition ability of the prepared CSPs with other CDMPC-based columns was also done. Our results were better than those reported by He et al. [2] using monolithic silica capillary with immobilized CDMPC through an intermolecular polycondensation or radical copolymerization (resolution value $<0.5$ for Tröger's base). Our resolution values were comparable with other works reported for silica (resolution values ranged 1.7-3.9) [42,43] or polyacrylamide-based (resolution values between 1.47 and 2.70) [9] monoliths coated with CDMPC under similar mobile phase conditions. In any case, it should be remarked that the developed CSP provided an incipient separation of basic compounds such as propranolol and mefloquin. In addition, a partial resolution of phenylalanine, a racemate very difficult to resolve, was found under RP conditions (see Fig. 4B), which demonstrates the high enantiorecognition capability of the prepared CDMPCcoated monolithic column.

\subsection{Column reproducibility and stability}

The reproducibility of preparation of columns photografted with META and posterior coating with CDMPC was also studied. The run-to-run repeatability of the monolithic CSP was evaluated from series of three injections of Tröger's base as representative analyte using the mobile phase conditions indicated in Fig. 3. RSD values of retention time and resolution for run-to-run injections were less than 0.4 and $3.3 \%$, respectively. To study the reproducibility of column preparation process, other two columns were prepared using the same process indicated above. The RSDs of retention time and resolution were below 1.9 and $10.6 \%$, respectively, which demonstrated a satisfactory reproducibility for column preparation.

In addition, the prepared monolithic chiral column was tested under different 
electrochromatographic conditions. No significant decrease of resolution factors and column deterioration were observed after the column was repeatedly used for multiple injections (above 100). This indicated the good stability of CDMPC-coated monolithic column.

\section{Conclusions}

In this work, novel methacrylate columns photografted with META and subsequently coated with the chiral selector CDMPC for enantiomeric separation in CEC have been prepared. The coating conditions (grafted META and CDMPC content) and mobile phase composition were optimized. The column that provided the best enantioseparation performance was achieved with $7.5 \mathrm{wt} \%$ META coated with $60 \mathrm{mg} \mathrm{mL}^{-1}$ CDMPC. A large number of quaternary groups photografted onto the polymeric monolith allowed to both incorporate CDMPC as chiral selector into the polymeric matrix as well as to provide a substantial EOF over a wide $\mathrm{pH}$ range. The prepared monolithic columns showed good enantioselectivity of several pairs of enantiomers under aqueous mobile phases. The reproducibility of developed columns was satisfactory, and they were stable under the adopted mobile phases. To the best knowledge of the authors, this works constitutes the first instance of CEC separation of chiral compounds on grafted polymeric monoliths with CDMPC as chiral selector. Due to the simple preparation of organic polymer-based monoliths jointly with the use of an easily implemented photografting process, the adopted strategy can be extended to the preparation of coated polysaccharide-derivatives or other chiral selector chemistries for enantioseparation in miniaturized chromatographic techniques.

\section{Acknowledgements}

The authors kindly acknowledge Projects CTQ2014-52765-R (MINECO of Spain and FEDER) and PROMETEO/2016/145 (Generalitat Valenciana); Consejo Nacional de 
Investigaciones Científicas y Técnicas (CONICET, Argentina) and Universidad Nacional de La Plata (UNLP, Argentina) for financial support.

\section{The authors have declared no conflict of interest.}

\section{References}

[1] Lv, C., Liu, Y., Mangelings, D., Vander Heyden, Y., Enantioselectivity of monolithic silica stationary phases immobilized with different concentrations cellulose tris $(3,5-$ dimethylphenylcarbamate), analyzed with different mobile phases in capillary electrochromatography. Electrophoresis 2011, 32, 2708-2717.

[2] He, C., Hendrickx, A., Mangelings, D., Smeyers-Verbeke, J., Heyden, Y.V., Monolithic silica capillary columns with immobilized cellulose tris(3,5dimethylphenylcarbamate) for enantiomer separations in CEC. Electrophoresis 2009, $30,3796-3803$.

[3] Lämmerhofer, M., Gargano, A., Monoliths with chiral surface functionalization for enantioselective capillary electrochromatography. J. Pharm. Biomed. Anal. 2010, 53, $1091-1123$.

[4] Fanali, C., Fanali, S., Chiral Separations using Miniaturized Techniques: State of the Art and Perspectives. Isr. J. Chem. 2016, 56, 958-967.

[5] Wu, Q., Sun, Y., Gao, J., Dong, S., Luo, G., Li, H., Zhao, L., Applications of hybrid organic-inorganic materials in chiral separation. TrAC Trends Anal Chem 2017, 95, $140-148$.

[6] Hernández-Borges, J., Aturki, Z., Rocco, A., Fanali, S., Recent applications in nanoliquid chromatography. J. Sep. Sci. 2007, 30, 1589-1610.

[7] Carrasco-Correa, E. J., Simo-Alfonso, E. F., Ramis-Ramos, G., Herrero-Martinez, J. M., Application of organic monolithic materials to enantioseparation in capillary 
separation techniques. Curr. Med. Chem. 2017, 24, 1-15.

[8] Zheng, J., Bragg, W., Hou, J., Lin, N., Chandrasekaran, S., Shamsi, S. A., Sulfated and sulfonated polysaccharide as chiral stationary phases for capillary electrochromatography and capillary electrochromatography-mass spectrometry. $J$. Chromatogr. A 2009, 1216, 857-872.

[9] Dong, X., Wu, R., Dong, J., Wu, M., Zhu, Y., Zou, H., Polyacrylamide-based monolithic capillary column with coating of cellulose tris(3,5-dimethylphenylcarbamate) for enantiomer separation in capillary electrochromatography. Electrophoresis 2008, 29, 919-927.

[10] Preinerstorfer, B., Lubda, D., Mucha, A., Kafarski, P., Lindner, W., Lämmerhofer, M., Stereoselective separations of chiral phosphinic acid pseudodipeptides by CEC using silica monoliths modified with an anion-exchange-type chiral selector. Electrophoresis 2006, 27, 4312-4320.

[11] Preinerstorfer, B., Lämmerhofer, M., Hoffmann, C. V., Lubda, D., Lindner, W., Deconvolution of electrokinetic and chromatographic contributions to solute migration in stereoselective ion-exchange capillary electrochromatography on monolithic silica capillary columns. J. Sep. Sci. 2008, 31, 3065-3078.

[12] Chankvetadze, B., Yamamoto, C., Tanaka, N., Nakanishi, K., Okamoto, Y., Highperformance liquid chromatographic enantioseparations on capillary columns containing monolithic silica modified with cellulose tris(3,5dimethyphenylcarbamate). J. Sep. Sci. 2004, 27, 905-911.

[13] Guerrouache, M., Millot, M.C., Carbonnier, B., Functionalization of macroporous organic polymer monolith based on succinimide ester reactivity for chiral capillary chromatography: a cyclodextrin click approach. Macromol. Rapid Commun. 2009, 30, 
$109-113$.

[14] Dixit, S., Lee, I. S., Park, J. H., Carbamoylated azithromycin incorporated zirconia hybrid monolith for enantioseparation of acidic chiral drugs using non-aqueous capillary electrochromatography. J. Chromatogr. A 2017, 1507, 132-140.

[15] Huang, B. Y., Chen, Y. C., Liu, C. Y., An insight into the mechanism of CEC separation of template analogues on a norepinephrine-imprinted monolith. J. Sep. Sci. 2011, 34, 2293-2300.

[16] Tian, Y., Zhang, L., Zeng, Z., Li, H., Calix[4] open-chain crown ether-modified, vinylfunctionalized hybrid silica monolith for capillary electrochromatography. Electrophoresis 2008, 29, 960-970.

[17] Hödl, H., Schmid, M. G., Gübitz, G., Chiral separation of amino acids and glycyl dipeptides by chiral ligand-exchange capillary electrophoresis comparing $\mathrm{Cu}(\mathrm{II})$, Co(II), Ni(II) and $\mathrm{Zn}(\mathrm{II})$ complexes of three different sugar acids. J. Chromatogr. A 2008, 1204, 210-218.

[18] Schmid, M. G., Gübitz, G., Enantioseparation by chromatographic and electromigration techniques using ligand-exchange as chiral separation principle. Anal. Bioanal. Chem. 2011, 400, 2305-2316.

[19] Al-Othman, Z. A., Al-Warthan, A., Ali, I., Advances in enantiomeric resolution on monolithic chiral stationary phases in liquid chromatography and electrochromatography. J. Sep. Sci. 2014, 37, 1033-1057.

[20] Chen, X., Qin, F., Liu, Y., Kong, L., Zou, H., Preparation of a positively charged cellulose derivative chiral stationary phase with copolymerization reaction for capillary electrochromatographic separation of enantiomers. Electrophoresis 2004, 25, $2817-2824$ 
[21] Meyring, M., Chankvetadze, B., Blaschke, G., Simultaneous separation and enantioseparation of thalidomide and its hydroxylated metabolites using highperformance liquid chromatography in common-size columns, capillary liquid chromatography and nonaqueous capillary electrochromatography. J. Chromatogr. A $2000,876,157-167$.

[22] Girod, M., Chankvetadze, B., Okamoto, Y., Blaschke, G., Highly efficient enantioseparations in non-aqueous capillary electrochromatography using cellulose tris(3,5-dichlorophenylcarbamate) as chiral stationary phase. J. Sep. Sci. 2001, 24, 2734.

[23] Rohr, T., Hilder, E. F., Donovan, J. J., Svec, F., Frechet, J. M. J., Photografting and the control of surface chemistry in three-dimensional porous polymer monoliths. Macromolecules 2003, 36, 1677-1684.

[24] Stachowiak, T. B., Rohr, T., Hilder, E. F., Peterson, D. S., Yi, M., Svec, F., Fréchet, J. M. J., Fabrication of porus polymer monoliths covalently attached to the walls of channels in plastic microdevices. Electrophoresis 2003, 24, 3689-3693.

[25] Pucci, V., Raggi, M. A., Svec, F., Fréchet, J. M. J., Monolithic columns with a gradient of functionalities prepared via photoinitiated grafting for separations using capillary electrochromatography. J. Sep. Sci. 2004, 27, 779-788.

[26] Eeltink, S., Hilder, E. F., Geiser, L., Svec, F., Fréchet, J. M. J., Rozing, G. P., Schoenmakers, P. J., Kok, W. T., Controlling the surface chemistry and chromatographic properties of methacrylate-ester-based monolithic capillary columns via photografting. J. Sep. Sci. 2007, 30, 407-413.

[27] Hilder, E. F., Svec, F., Fréchet, J. M. J., Shielded stationary phases based on porous polymer monoliths for the capillary electrochromatography of highly basic 
biomolecules. Anal. Chem. 2004, 76, 3887-3892.

[28] Connolly, D., O`Shea, V., Clark, P., O`Connor, B., Paull, B., Evaluation of photografted charged sites within polymer monoliths in capillary columns using contactless conductivity detection. J. Sep. Sci. 2007, 30, 3060-3068.

[29] Ling, F., Brahmachary, E., Xu, M., Svec, F., Fréchet, J. M. J., Polymer-bound cellulose phenylcarbamate derivatives as chiral stationary phases for enantioselective HPLC. J. Sep. Sci. 2003, 26, 1337-1346.

[30] Castells, C. B., Carr, P. W., Coated zirconia as a chiral stationary phase for HPLC. Anal. Chem. 1999, 71, 3013-3021.

[31] Peters, E. C., Petro, M., Svec, F., Fréchet, J. M. J., Molded rigid polymer monoliths as separation media for capillary electrochromatography. 1. Fine control of porous properties and surface chemistry. Anal. Chem. 1998, 70, 2288-2295.

[32] Peters, E. C., Petro, M., Svec, F., Fréchet, J. M. J., Molded rigid polymer monoliths as separation media for capillary electrochromatography. Anal. Chem. 1997, 69, 36463649 .

[33] Carrasco-Correa, E. J., Ramis-Ramos, G., Herrero-Martínez, J. M., Methacrylate monolithic columns functionalized with epinephrine for capillary electrochromatography applications. J. Chromatogr. A 2013, 1298, 61-67.

[34] Carrasco-Correa, E. J., Ramis-Ramos, G., Herrero-Martínez, J. M., Evaluation of 2,3epoxypropyl groups and functionalization yield in glycidyl methacylate monoliths using gas chromatography, J. Chromatogr. A 2014, 1379, 100-105.

[35] Stachowiak, T. B., Svec, F., Fréchet, J. M. J., Patternable protein resistant surfaces for multifunctional microfluidic devices via surface hydrophilization of porous polymer monoliths using photografting. Chem. Mater. 2006, 18, 5950-5957. 
[36] Currivan, S., Connolly, D., Paull, B., Stepped gradients on polymeric monolithic columns by photoinitiated grafting. J. Sep. Sci. 2015, 38, 3795-3802.

[37] Connolly, D., Paull, B., High-performance separation of small inorganic anions on a methacrylate-based polymer monolith grafted with [2(methacryloyloxy)ethyl] trimethylammonium chloride. J. Sep. Sci. 2009, 32, 2653-2658.

[38] Vonk, R. J., Wouters, S., Barcaru, A., Vivó-Truyols, G., Eeltink, S., de Koning, L. J., Schoenmakers, P. J., Post-polymerization photografting on methacrylate-based monoliths for separation of intact proteins and protein digests with comprehensive two-dimensional liquid chromatography hyphenated with high-resolution mass spectrometry. Anal. Bioanal. Chem. 2015, 3817-3829.

[39] Chankvetadze, B., Kubota, T., Ikai, T., Yamamoto, C., Tanaka, N., Nakanishi, K., Okamoto, Y., High-performance liquid chromatographic enantioseparations on capillary colums containing crosslinked polysaccharide phenylcarbamate derivatives attached to monolithic silica. J. Sep. Sci. 2006, 29, 1988-1995.

[40] Fanali, S., D’Orazio, G., Lomsadze, K., Chankvetadze, B., Enantioseparations with cellulose tris(3-chloro-4-methylphenylcarbamate) in nano-liquid chromatography and capillary electrochromatography. J. Chromatogr. B Anal. Technol. Biomed. Life Sci. $2008,875,296-303$.

[41] Qin, F., Liu, Y., Chen, X., Kong, L., Zou, H., Capillary electrochromatographic separation of enantiomers under aqueous mobile phases on a covalently bonded cellulose derivative chiral stationary phase. Electrophoresis 2005, 26, 3921-3929.

[42] Ou, J., Lin, H., Tang, S., Zhang, Z., Dong, J., Zou, H., Hybrid monolithic columns coated with cellulose tris(3,5-dimethylphenyl-carbamate) for enantioseparations in capillary electrochromatography and capillary liquid chromatography. J. Chromatogr. 
A 2012, 1269, 372-378.

[43] Qin, F., Xie, C., Feng, S., Ou, J., Kong, L., Ye, M., Zou, H., Monolithic silica capillary column with coated cellulose tris (3,5-dimethylphenyl-carbamate) for capillary electrochromatographic separation of enantiomers. Electrophoresis 2006, 27, 10501059.

\section{Figure captions}

Fig. 1 SEM micrographs of (A) poly(GMA-co-EDMA) monolith and (B) poly(GMA-coEDMA) photografted with 7.5\% META and coated with $60 \mathrm{mg} \mathrm{mL}^{-1}$ CDMPC. Magnification at $14000 \times$.
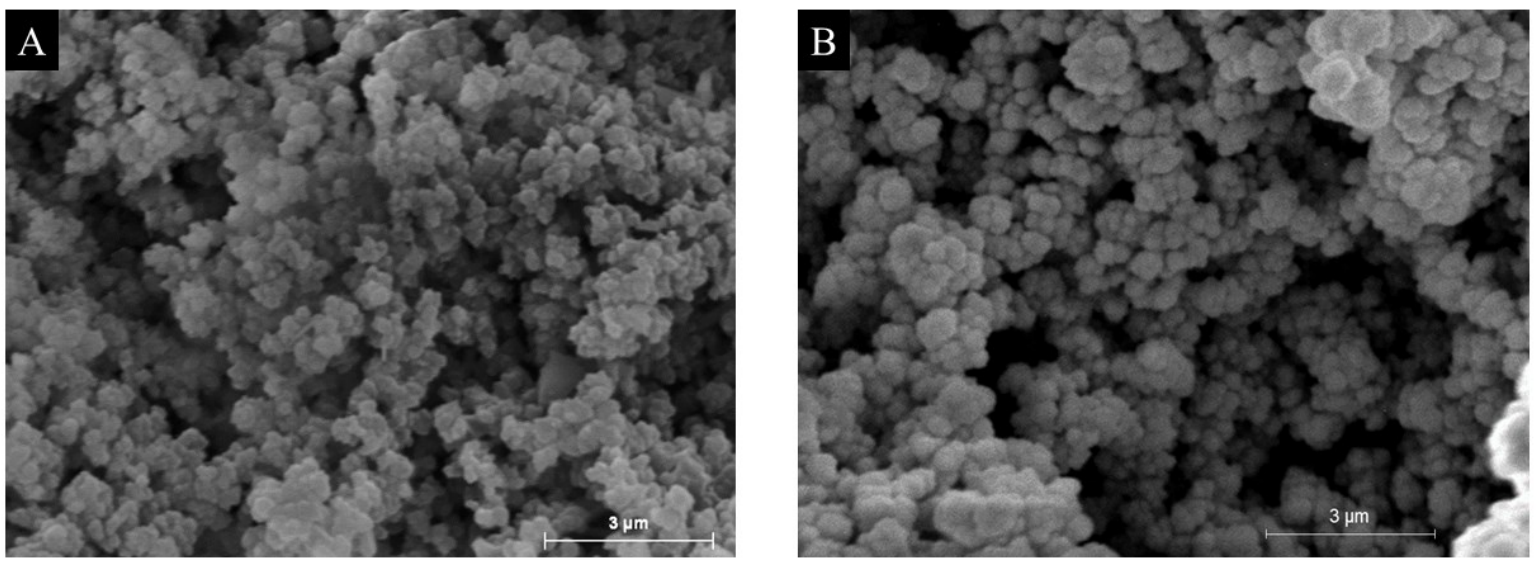

Fig. 1 . Echevarría, R.N. et al.

This article is protected by copyright. All rights reserved. 
Fig. 2 CEC separations of Tröger's base on CDMPC-coated monolithic columns grafted with different META content. Experimental conditions: Monolithic columns coated with $60 \mathrm{mg}$ $\mathrm{mL}^{-1}$ of CDMPC; other conditions as those described in Table 1.

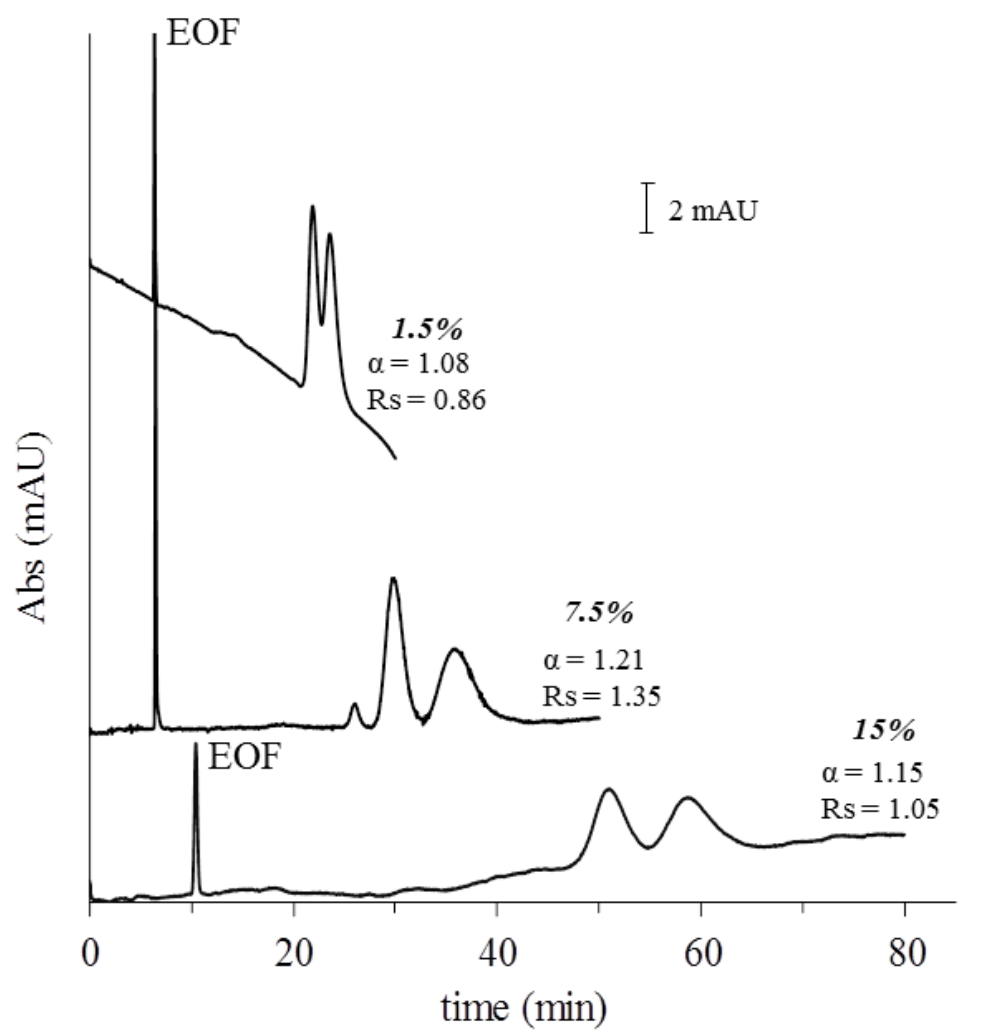

Fig. 2. Echevarría, R.N. et al.

This article is protected by copyright. All rights reserved. 
Fig. 3 Influence of ACN content in mobile phase on enantioseparation of Tröger's base. Other conditions as those described in Table 1.

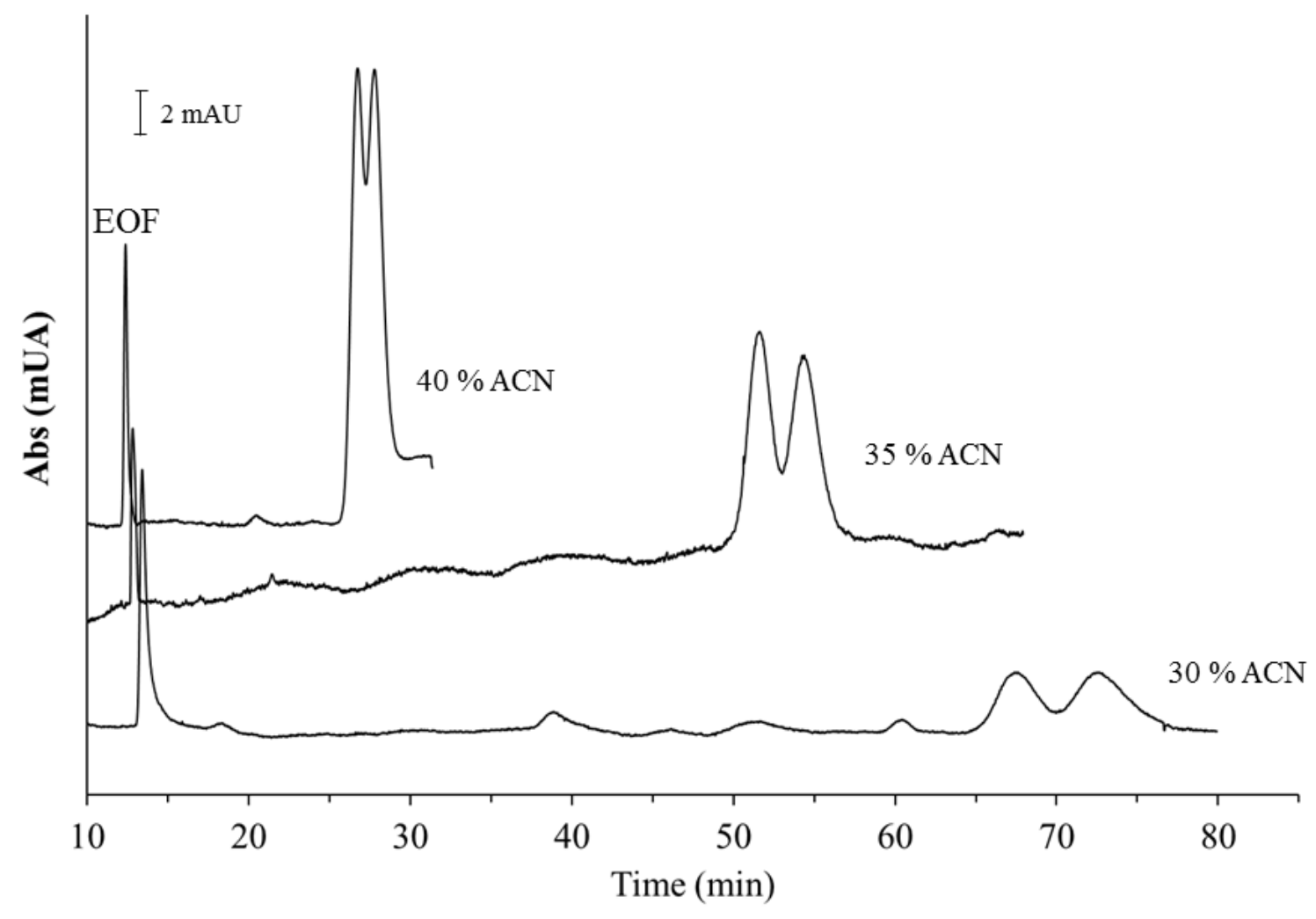

Fig. 3. Echevarría, R.N. et al.

This article is protected by copyright. All rights reserved. 
Fig. 4 Electrochromatograms of (A) Tröger's base and (B) phenylalanine. Experimental conditions for each solute as indicated in Table 2.

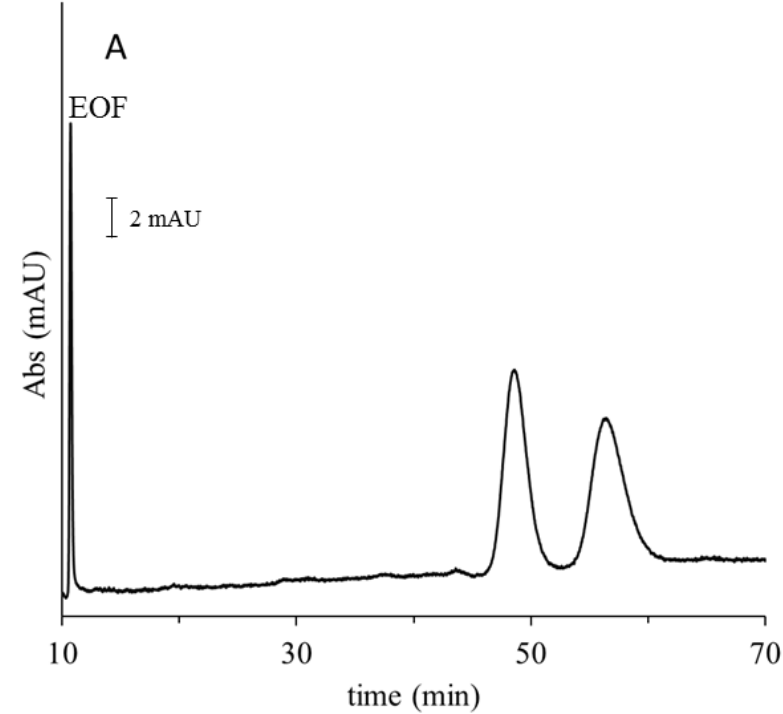

Table 1 CEC enantioseparation for Tröger's base on the photografted-META column coated with different amounts of CDMPC ${ }^{\mathrm{a}}$.

\begin{tabular}{ccccccc}
\hline CDMPC $\left(\mathbf{m g ~ m L}^{-\mathbf{1}}\right)$ & $\boldsymbol{k}_{\boldsymbol{1}}{ }^{*}$ & $\boldsymbol{k}_{\boldsymbol{2}}{ }^{*}$ & $\boldsymbol{\alpha}$ & $\boldsymbol{R} \boldsymbol{s}$ & $\boldsymbol{N}_{\boldsymbol{1}}{ }^{*}\left(\boldsymbol{m}^{-1}\right)$ & $\boldsymbol{N}_{\mathbf{2}}{ }^{*}\left(\boldsymbol{m}^{-1}\right)$ \\
\hline 0 & 2.73 & 2.73 & 1.00 & 0.00 & 30,700 & 30,700 \\
6 & 3.13 & 3.20 & 1.02 & $<0.50$ & 10,600 & 7,100 \\
15 & 3.71 & 3.95 & 1.06 & $<0.50$ & 8,400 & 4,300 \\
40 & 3.26 & 3.50 & 1.07 & 0.53 & 7,200 & 5,100 \\
60 & 2.43 & 2.69 & 1.11 & 0.86 & 5,400 & 4,200 \\
\hline
\end{tabular}

${ }^{a}$ Experimental conditions: column, $33.5(25 \mathrm{~cm}) \times 100 \mu \mathrm{m}$ i.d.; photografting conditions: first step, $5 \mathrm{wt} \%$ BP for $10 \mathrm{~min}$; second step, irradiation with $1.5 \mathrm{wt} \%$ META content for $10 \mathrm{~min}$; mobile phase, 35/65 (v/v) $\mathrm{ACN} / \mathrm{H}_{2} \mathrm{O} 5 \mathrm{mM}$ phosphate buffer, $\mathrm{pH} 7.0$ ); injection, $5 \mathrm{kV} \times 3 \mathrm{~s}$, applied voltage, $-10 \mathrm{kV}$; detection at $214 \mathrm{~nm}$.

"The subscripts " 1 " and " 2 " designate the $1^{\text {st }}$ and $2^{\text {nd }}$ eluted peak of the enantiomer pair, respectively.

This article is protected by copyright. All rights reserved. 
Table 2. CEC enantiomer separation of various test racemic compounds on the META photografted column coated with CDMPC ${ }^{\mathrm{a}}$.

\begin{tabular}{cccccccc}
\hline Analyte & $\begin{array}{c}\text { Mobile } \\
\text { phase }\end{array}$ & $\boldsymbol{k}_{\boldsymbol{1}}{ }^{*}$ & $\boldsymbol{k}_{\boldsymbol{2}}{ }^{*}$ & $\boldsymbol{\alpha}$ & $\boldsymbol{R} \boldsymbol{c}$ & $\boldsymbol{N}_{\boldsymbol{1}}{ }^{*}\left(\boldsymbol{m}^{-1}\right)$ & $\boldsymbol{N}_{2}{ }^{*}\left(\boldsymbol{m}^{-1}\right)$ \\
\hline Trans-stilbene oxide & $\mathrm{b}$ & 1.36 & 1.55 & 1.11 & 1.29 & 23,500 & 6,400 \\
Benzoin & $\mathrm{b}$ & 3.18 & 3.18 & 1.04 & 0.59 & 20,200 & 16,000 \\
2-phenyl-1-propanol & $\mathrm{a}$ & 3.06 & 6.06 & 1.87 & 4.30 & 6,700 & 6,900 \\
Tröger's base & $\mathrm{b}$ & 3.54 & 4.27 & 1.20 & 1.77 & 11,100 & 8,900 \\
Mefloquin & $\mathrm{a}$ & 4.74 & 4.98 & 1.05 & $<0.50$ & 3,100 & 2,500 \\
Propanolol & $\mathrm{b}$ & 7.28 & 7.58 & 1.04 & $<0.50$ & 20,700 & 3,700 \\
Ibuprofen & $\mathrm{a}$ & 0.41 & 0.96 & 1.38 & 1.61 & 1,600 & 1,600 \\
Naproxen & $\mathrm{b}$ & 1.91 & 2.07 & 1.05 & 0.63 & 11,600 & 11,200 \\
Phenylalanine & $\mathrm{a}$ & 4.62 & 4.72 & 1.02 & 0.59 & 63,000 & 110,300 \\
\hline
\end{tabular}

${ }^{a}$ Experimental conditions: column, $33.5(25 \mathrm{~cm}) \times 100 \mu \mathrm{m}$ i.d.; photografting conditions: first step, $5 \mathrm{wt} \%$ $\mathrm{BP}$ for $10 \mathrm{~min}$; second step, irradiation with $7.5 \mathrm{wt} \%$ META content for $10 \mathrm{~min}$; CDMPC coating: $60 \mathrm{~min}$ at $60 \mathrm{mg} \mathrm{mL}^{-1}$; mobile phase, 35/65 (v/v) ACN/ $\mathrm{H}_{2} \mathrm{O}$ containing: (a) $5 \mathrm{mM}$ acetic/acetate buffer, $\mathrm{pH} 3.6$ or (b) $5 \mathrm{mM}$ phosphate buffer, $\mathrm{pH} 7.0$; injection, $5 \mathrm{kV} \times 3 \mathrm{~s}$, applied voltage, $-10 \mathrm{kV}$; detection at $214 \mathrm{~nm}$.

"The subscripts " 1 " and " 2 " designate the $1^{\text {st }}$ and $2^{\text {nd }}$ eluted peak $\theta$ f the enantiomer pair, respectively.

This article is protected by copyright. All rights reserved. 